\title{
Imaging features of soft tissue epithelioid angiosarcoma in the lower extremity: A case report
}

\author{
LULU LV ${ }^{1}$, PENG XU ${ }^{2}$, YIBING SHI ${ }^{1}$, JINGMING HAO $^{1}$, CHUNAI HU $^{1}$ and BAOZHONG ZHAO ${ }^{1}$ \\ ${ }^{1}$ Department of Computed Tomography and Magnetic Resonance Imaging, Xuzhou Central Hospital, Xuzhou, Jiangsu 221009; \\ ${ }^{2}$ Department of Radiology, The Affiliated Hospital of Xuzhou Medical College, Xuzhou, Jiangsu 221006, P.R. China
}

Received February 1, 2015; Accepted December 10, 2015

DOI: $10.3892 / 01.2016 .4407$

\begin{abstract}
Epithelioid angiosarcomas are extremely rare malignant tumors formed from endothelial cells. The majority of studies reporting these tumors have been concerned with the clinical and pathological aspects, with limited reporting of radiological diagnosis. The aim of the present study was to provide a reference to improve understanding of diagnosis, treatment choice and prognosis assessment of epithelioid angiosarcoma. The current study reports the case of a 44-year-old woman with epithelioid angiosarcoma located in the deep soft tissue of the lower extremities. Physical examination of the right thigh revealed a palpable hard mass and movement was clearly restricted and painful. X-ray, computed tomography (CT) scans and magnetic resonance imaging (MRI) were used to evaluate the imaging features of the tumor. Using X-ray and CT scanning, an inhomogenous tumor with osteolytic osseous destruction was observed. MRI revealed that the bordering skeletal muscles were infiltrated. The patient was treated with palliative surgery and chemotherapy, but succumbed to disease 1 year later.
\end{abstract}

\section{Introduction}

Angiosarcoma accounts for $2 \%$ of soft tissue sarcomas (1). Epithelioid angiosarcoma is a rare type of angiosarcoma that was first reported by $\mathrm{Ng}$ et al in 1996 (2). Epithelioid angiosarcoma occurs in various sites, including the skin, head and neck, lungs, breasts, adrenal glands, bones and deep soft tissue in the extremities (3-8). Epithelioid angioma has a male predilection and typically occurs in adults, with the highest incidence being in the seventh decade of life (4). Treatment modalities include surgical resection of the primary tumor,

Correspondence to: Ms. Yibing Shi, Department of Computed Tomography and Magnetic Resonance Imaging, Xuzhou Central Hospital, 199 Jiefang South Road, Xuzhou, Jiangsu 221009, P.R. China

Email: sybxuzhou@163.com

Key words: soft tissue tumors, epithelioid angiosarcoma, computed tomography, magnetic resonance imaging radiation therapy and chemotherapy (4-6). Within 2-3 years of diagnosis, $\sim 50 \%$ of patient succumb to disease, however, $20-30 \%$ of patients are free of disease $(4,9)$. Advanced age, increased tumor size and a retroperitoneal primary site are considered to be adverse prognostic factors (9). Although pathological examination is the gold standard of diagnosis, radiological studies may provide assistance for determining the treatment choice and assessing the prognosis (10). Magnetic resonance imaging (MRI) reveals the infiltration of the lesion, and computed tomography (CT) angiography (CTA) reveals the involvement of the arteries in the lower extremities, which determines whether or not thrombosis is present $(3,4)$. The current study presents the imaging analysis of a patient with epithelioid angiosarcoma that occurred in the deep soft tissue of the lower extremities. Written informed consent was obtained from the patient.

\section{Case report}

A 44-year-old woman presented at the Department of Radiology, The Affiliated Hospital of Xuzhou Medical College (Jiangsu, China) on September 16, 2012 with soreness in the medial side of the right thigh, which had developed over 2 months. Almost 1 month later, a mass had evolved at the painful site; however, the pain was not associated with exertion. A physical examination revealed an increased temperature on the medial side, at the mid-shaft of the femur. The mass was $\sim 5.0 \times 4.0 \mathrm{~cm}$ in size, palpable, hard, red and swollen, although there was no evident varicosity. The mass had undefined margins and was not easy to move. A vascular murmur was also identified, but no notable sensory loss on the skin and good peripheral circulation were identified.

Following an X-ray, multiple osteolytic lesions involving the cortex and medullary cavity, with defined margins, were identified in the right femur (Fig. 1). The CT scan revealed that the lesions were centered in the medial aspect of the quadriceps muscle group, affecting the vastus medialis and intermedius muscles of the soft tissue window, with decreased heterogeneous density and a blurred intermuscular plane (Fig. 2A). The CT scan revealed the presence of osteolytic lesions on the right femoral cortex of the bone window, the inside of which had a soft tissue density (Fig. 2B). The findings of the MRI scan were as follows: T1 weighted image (WI) sequence identified a mass with a low signal that was located in the adductor group 


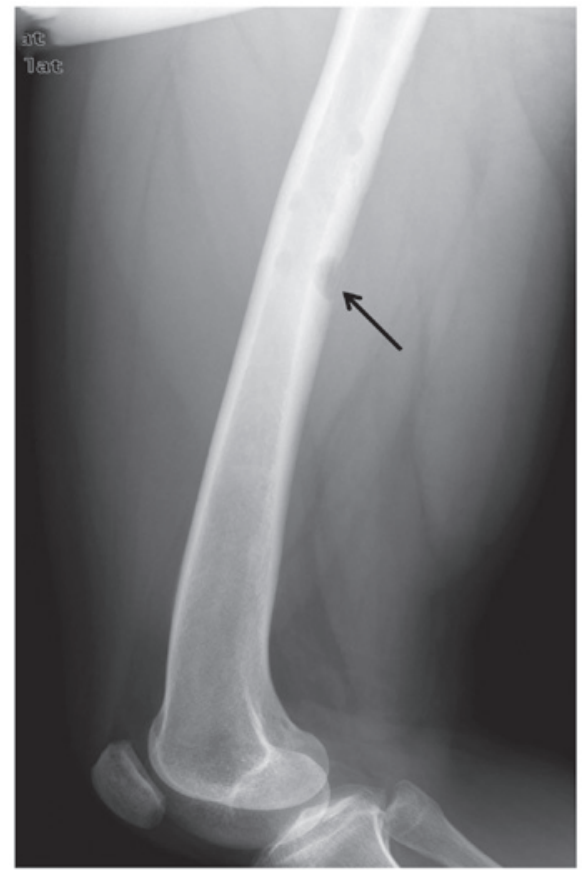

Figure 1. X-ray lateral imaging of the lower extremity of the paitent. Arrow indicates multiple osteolytic lesions in the right proximal femur.
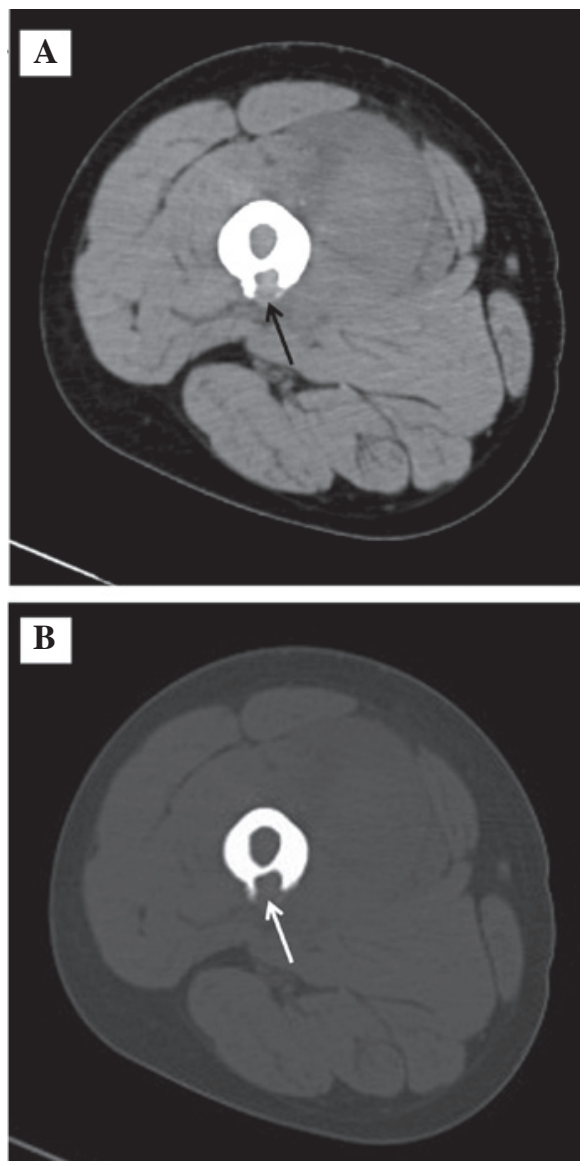

Figure 2. CT scans of the lower extremities of the patient. (A) Axial CT scan, soft tissue window. The CT scan identified that the lesion was centered in the medial aspect of the quadriceps muscle group, affecting the vastus medialis and intermedius muscles on the soft tissue window, with decreased heterogeneous density and blurred intermuscular plane, identified by the arrow. (B) Axial CT scan, bone window. CT scan demostrates osteolytic lesions on the right femoral cortex on the bone window, with the soft tissue density inside it, as identified by the arrow. CT, computed tomography. of the right femur (Fig. 3A); and the T2WI sequence identified a mass with a slightly increased signal that was surrounded by a low-signal capsule, and adjacent muscles demonstrated a large patch with a slightly high signal with undefined margins (Fig. 3B and C); the diffusion weighted imaging sequence revealed a heterogeneous high signal (Fig. 3D). An enhanced MRI scan demonstrated a heterogeneous enhancement of the mass, possessing undefined demarcation with adjacent normal muscle tissue and non-enhanced necrosis inside the lesion (Fig. 3E).

The mass was located in the adductor group of the right thigh, with an intact capsule and sufficient blood supply. A biopsy of the lesion was performed. Hematoma and necrosis were present inside the tumor mass. Hematoxylin and eosin (Nanjing Jiancheng Bioengineering Institute, Nanjing, China) staining revealed epithelioid tumor cells that were oval or irregular in shape with abundant eosinophilic cytoplasms, unequal and vacuolar nuclei and certain notable nucleoli (Fig. 4A). Immunohistochemistry (Nanjing Jiancheng Bioengineering Institute, Nanjing, China) revealed the presence of cluster of differentiation (CD)-31 (Fig. 4B), CD34, 2-keto-6-phosphate-D-gluconic acid, factor VIII and ki-67 $(80 \%)$ in the carcinoma cells. The pathological diagnosis was epithelioid angiosarcoma.

Since the patient did not undergo amputation surgery, palliative surgery was adopted and the tumor was excised, followed by chemotherapy. Thrombosis was observed in the femoral artery of the leg following the surgery. In total, 6 months subsequent to surgery, multiple osteolytic lesions on the right ribs and metastases on the lungs were located by a CT scan (Fig. 5). By the 1-year follow-up, the patient had succumbed to the carcinoma.

\section{Discussion}

The majority of epithelioid angiosarcomas occur in males and the incidence peaks at $\sim 70$ years of age (9). The clinical manifestations of epithelioid angiosarcomas vary depending on the sites of the lesions (10). A tumor diagnosis must be corroborated by immunohistochemical analysis. Classical tumor cells reveal the presence of CD31, CD34 and factor VIII (11). In the early stages, epithelioid angiosarcomas are able to metastasize to lymph nodes and parenchymal organs, particularly the lung, bone and soft tissue $(1,3)$. In total, $>50 \%$ of patients succumb to the disease within 2-3 years of a confirmed diagnosis $(4,9)$. Treatment varies according to the patient, but radical excision combined with radiotherapy is generally considered to be the routine method $(12,13)$. According to certain studies, lesion containment and limb preservation using radiotherapy may be beneficial to primary or relapsed tumors; however, the survival rate is not increased $(4,7,14)$.

Imaging features of epithelioid angiosarcoma in the deep soft tissue of the lower extremities are usually identified by X-ray, CT and MRI. X-ray usually reveals that the soft tissue is swollen with osteolytic destruction on the adjacent cortex, while the CT scan demonstrates that the lesions manifest as soft tissue masses with undefined margins and blurred adjacent intermuscular planes. CT bone window scans are superior to X-rays, due to the improved evaluation of bone destruction. 

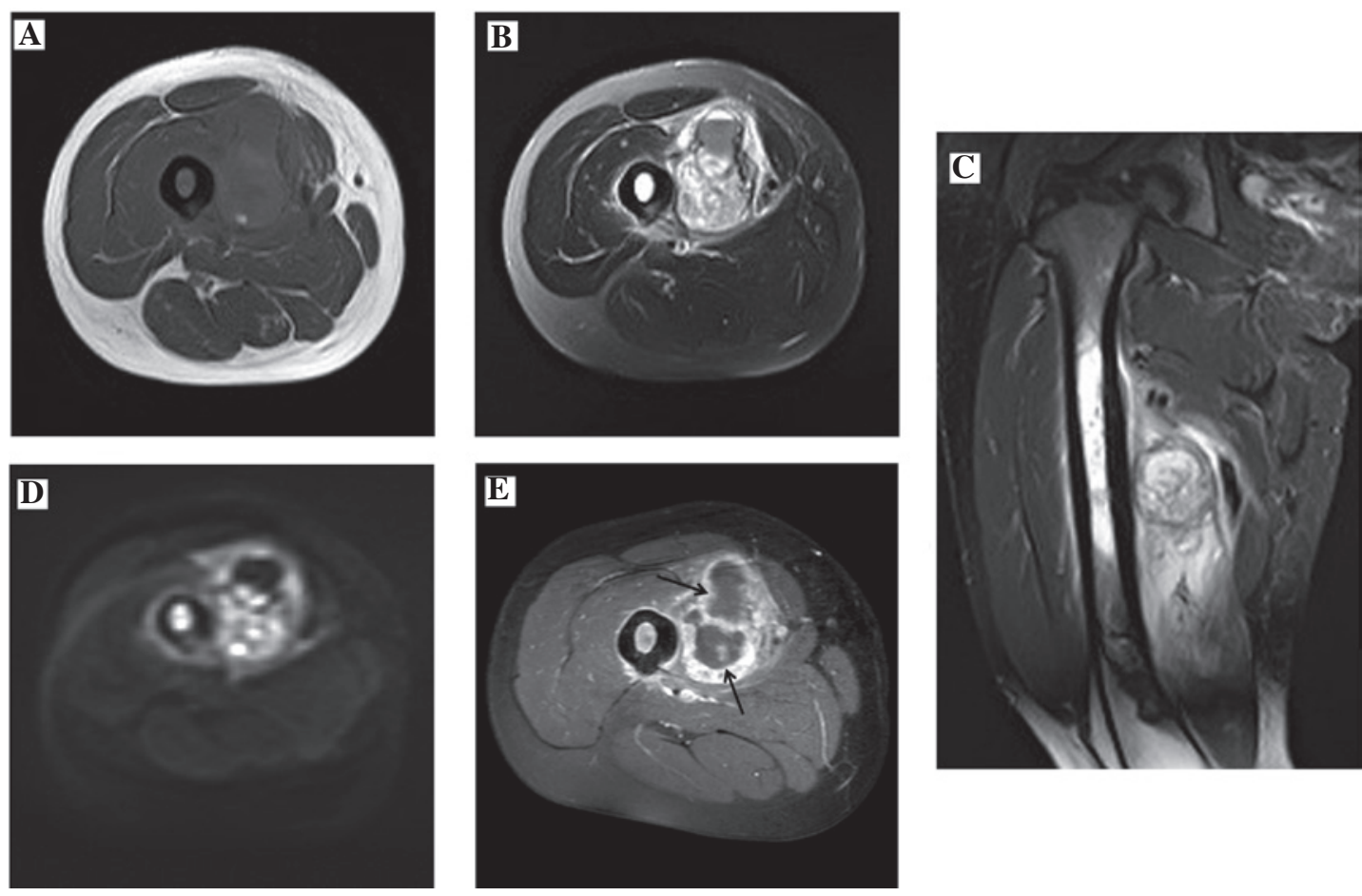

Figure 3. MRI scans of the mass located in the lower extremities of the female patient. (A) Routine axial T1WI scan. A mass with an equally low signal mixed with a mottled high signal was located in the adductor group of the right femur. (B) Routine axial T2WI scan. (C) T2WI MRI coronal fat suppression scan. A mass with a slightly high signal that was surrounded by a low-signal capsule was identified, and the adjacent muscle demonstrated a large patch with a slightly increased signal with undefined margins. (D) Axial DWI scan. DWI demonstrated a heterogeneous high signal. (E) Axial T1WI enhancement scan. A heterogeneous enhancement of the mass was revealed, with undefined demarcation with adjacent normal muscle tissue and non-enhanced necrosis inside the lesion, as identified by the arrow. MRI; magnetic resonance imaging; WI, weighted image; DWI, diffusion weighted imaging.
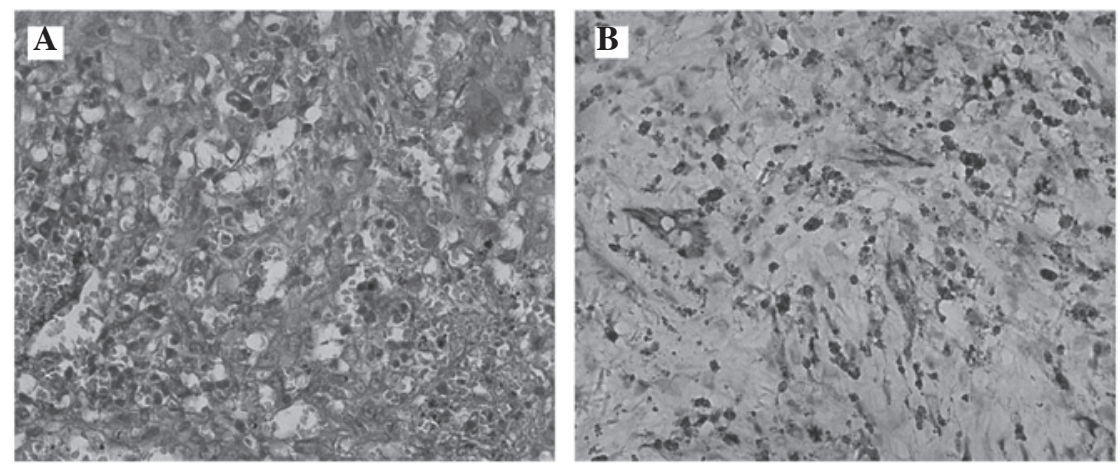

Figure 4. Histopathological and immunohistochemical analysis of the epithelioid angiosarcoma. (A) Hemotoxylin and eosin staining revealed epithelioid tumor cells with abundant eosinophilic cytoplasms, oval or irregular in shape, with unequal and vacuolar nuclei and notable nucleoli. (B) Cells expressed cluster of differentiation 31, as indicated by the black areas. Magnification x200.

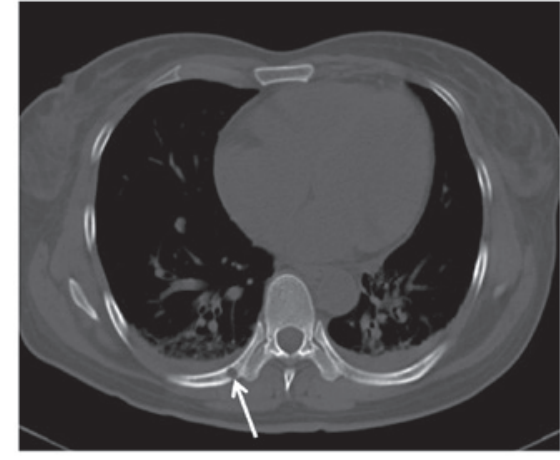

Figure 5. Chest computed tomography scan, bone window, of the female patient 6 months subsequent to surgery. Multiple osteolytic lesions on the right ribs were identified.
Lower extremity CTAs aid the evaluation of the association between masses and arteries, identification of the origin of the tumor and selection of a surgical approach (10). MRI scans allow evaluation of the tumor infiltration and internal structure. In the present study, the epithelioid angiosarcoma mass manifested as a mottled, increased signal on T1WI MRI, suggesting an abundant blood supply and predisposition to internal hemorrhage (4). An enhanced MRI scan may reveal the infiltration level of a lesion. In the current study, an enhanced MRI scan of the lesion demonstrated that there was significant enhancement of the solid component of the tumor, and the enhancement region was much more extensive compared with the routine MRI scan. This provided information concerning the amount of radical excision to be performed by the surgeon. 
Non-enhanced regions inside a tumor, as demonstrated by an enhanced MRI scan, suggest rapid growth with ischemia and local necrosis (15).

In order to provide an accurate prognosis and correct treatment of epithelioid angiosarcomas, the tumors are classified into two categories. Primary tumors are those that have been diagnosed for the first time without metastasis and are generally resectable. Developing tumors are those that are diagnosed for the first time with metastases or those that are unresectable (16). It has been reported that the average survival time for a developing tumor is $\sim 7.3$ months (16).

It is important to eliminate differential diagnoses of epithelioid angiosarcomas when diagnosing soft tissue masses in the lower extremities. Hemangiomas in the lower extremities are mainly plexiform cavernous hemangiomas. On T1WI MRI, these demonstrate an equal or slightly increased signal, while T2WI MRI reveals a high signal without bone destruction on adjacent structures, along with defined margins with adjacent soft tissue. Fibrosarcomas mainly occur in the elderly, and these exhibit relatively low signals on T1WI and T2WI MRI. The enhancement of fibrosarcomas is generally not significant compared with epithelioid angiosarcomas when using an MRI enhancement scan. Metastasis generally occurs in elderly patients, who are usually predisposed to pathological fractures. Metastases are not challenging to identify based on clinical symptoms and primary lesions. In clinical practice, if a pampiniform abnormal density signal is identified in tubular bones with evident enhancement on a MRI scan, along with adjacent bone destruction, it is crucial for the clinician to consider a diagnosis of epithelioid angiosarcoma. Phlebolith-like calcifications or hemorrhaging also supports this diagnosis. However, the gold standard for diagnosing epithelioid angiosarcomas remains pathological examination (10).

In summary, there are specific imaging features that can be used in the diagnosis of epithelioid angiosarcomas of the soft tissue of the lower extremities. The present study aimed to improve the understanding of this disease for radiologists. Together, X-ray, CT and MRI scans may be effective in diagnosing epithelioid angiosarcomas by eliminating differential diagnosis of other soft tissue masses of the lower extremities.

\section{References}

1. Cafiero F, Gipponi M, Peressini A, Queirolo P, Bertoglio S, Comandini D, Percivale P, Sertoli MR and Badellino F: Radiation-associated angiosarcoma: Diagnostic and therapeutic implications - two case reports and a review of the literature. Cancer 77: 2496-2502, 1996.

2. Ng WK, Collins RJ, Law D and Gwi E: Cutaneous epithelioid angiosarcoma: A potential diagnostic trap for cytopathologists. Diagn Cytopathol 16: 160-166, discussion 166-167, 1997.

3. Asmane I, Litique V, Heymann S, Marcellin L, Métivier AC, Duclos B, Bergerat JP and Kurtz JE: Adriamycin, cisplatin, ifosfamide and paclitaxel combination as front-line chemotherapy for locally advanced and metastatic angiosarcoma. Analysis of three case reports and review of the literature. Anticancer Res 28 (5B): 3041-3045, 2008.

4. Deshpande V, Rosenberg AE, O'Connell JX and Nielsen GP: Epithelioid angiosarcoma of the bone: A series of 10 cases. Am J Surg Pathol 27: 709-716, 2003.

5. Lund L and Amre R: Epithelioid angiosarcoma involving the lungs. Arch Pathol Lab Med 129: e7-e10, 2005.

6. Goldblum JR and Rice TW: Epithelioid angiosarcoma of the pulmonary artery. Hum Pathol 26: 1275-1277, 1995.

7. Ghanem N, Riede U, Uhrmeister P, Weigang E and Altehoefer C: Epithelioid angiosarcoma of the aorta. Vasa 31: 269-273, 2002.

8. Macías-Martínez V, Murrieta-Tiburcio L, Molina-Cárdenas H and Domínguez-Malagón H: Epithelioid angiosarcoma of the breast. Clinicopathological, immunohistochemical, and ultrastructural study of a case. Am J Surg Pathol 21: 599-604, 1997.

9. Meis-Kindblom JM and Kindblom LG: Angiosarcoma of soft tissue: A study of 80 cases. Am J Surg Pathol 22: 683-697, 1998.

10. Hart J and Mandavilli S: Epithelioid angiosarcoma: A brief diagnostic review and differential diagnosis. Arch Pathol Lab Med 135: 268-272, 2011.

11. Callister MD, Ballo MT, Pisters PW, Patel SR, Feig BW, Pollock RE, Benjamin RS and Zagars GK: Epithelioid sarcoma: Results of conservative surgery and radiotherapy. Int J Radiat Oncol Biol Phys 51: 384-391, 2001.

12. Lăzureanu C, Baderca F, Burlacu O and Nicodin A: Soft tissue epithelioid angiosarcoma. Rom J Morphol Embryol 51: 787-792, 2010.

13. Lydiatt WM, Shaha AR and Shah JP: Angiosarcoma of the head and neck. Am J Surg 168: 451-454, 1994.

14. Mendeszoon MJ, Mendeszoon ER Jr, Rasmussen S, Bell J and Harris SY: Epithelioid angiosarcoma of the talus. J Foot Ankle Surg 50: 87-92, 2011.

15. Bürk J, Gerlach U, Baumann T, Langer M and Winterer JT: Epithelioid angiosarcoma of the scapula. In Vivo 24: 783-786, 2010

16. Abraham JA, Hornicek FJ, Kaufman AM, Harmon DC, Springfield DS, Raskin KA, Mankin HJ, Kirsch DG, Rosenberg AE, Nielsen GP, et al: Treatment and outcome of 82 patients with angiosarcoma. Ann Surg Oncol 14: 1953-1967, 2007. 